\begin{tabular}{|c|c|c|c|c|c|c|}
\hline \multirow{4}{*}{ Impact Factor: } & ISRA (India) & $=3.117$ & SIS (USA) & $=0.912$ & ICV (Poland) & $=6.630$ \\
\hline & ISI (Dubai, UAE & $=0.829$ & РИНЦ (Russia & $=0.156$ & PIF (India) & $=1.940$ \\
\hline & GIF (Australia) & $=0.564$ & ESJI (KZ) & $=8.716$ & IBI (India) & $=4.260$ \\
\hline & JIF & $=1.500$ & SJIF (Morocce & $=5.667$ & OAJI (USA) & $=0.350$ \\
\hline
\end{tabular}

\section{SOI: $\underline{1.1 / \mathrm{TAS}}$ DOI: $\underline{10.15863 / \mathrm{TAS}}$ International Scientific Journal Theoretical \& Applied Science}

p-ISSN: 2308-4944 (print) e-ISSN: 2409-0085 (online)

Year: 2019 Issue: $05 \quad$ Volume: 73

Published: $30.05 .2019 \quad$ http://T-Science.org

SECTION 2. Applied mathematics.

Mathematical modeling
QR - Issue

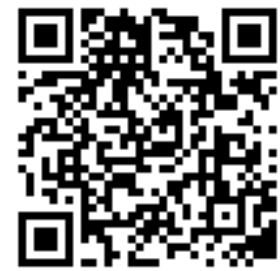

Corresponding Member of International Academy of Theoretical and Applied Sciences (USA), Candidate of physics and mathematical sciences, Department «Information technologies and automation», Professor, Noncommercial joint-stock company "Kazakh national agrarian university", Kazakhstan sapagtu@mail.ru

A. Agaev

Master student, Noncommercial joint-stock company "Kazakh national agrarian university" agaevbz5@gmail.com

\title{
A GIVING REALISM TO THE PROPERTY VALUES OF THE CEREAL CROPS IN THE MODEL $\Lambda$-SAMPLE
}

Abstract: The article describes the application of the IM PCA: $\left(\boldsymbol{C}_{66}, \Lambda_{66}\right)=>\left(R_{6,6}, U^{(t)}{ }_{20,6}, Y^{(t)}{ }_{20,6}=U^{(t)}{ }_{20,6} \Lambda^{1 / 2}{ }_{66}\right.$, $\left.I_{6,6}=Z^{(t)}{ }_{20,6}=Y^{(t)}{ }_{20,6} C_{6,6}^{T}\right), t=1, \ldots, k_{t}<\infty$. The sample $U_{20,6}$ of $u$-variables has a correlation matrix $(1 / 20) U^{(t) T}{ }_{20,6} U^{(t)}{ }_{20,6}=$ $\operatorname{diag}(1, \ldots, 1)$, the sample of $y$-variables $Y_{20,6}=U_{20,6} \Lambda^{1 / 2}{ }_{66}$ : covariance matrix $\Lambda_{n n}=\operatorname{diag}\left(\lambda_{1}, \lambda_{2}, \ldots, \lambda_{6}\right)$, the sample of $z$ variables $Z^{(t)}{ }_{20,6}=Y^{(t)}{ }_{20,6} C_{6,6}^{T}=U^{(t)}{ }_{20,6} \Lambda^{1 / 2}{ }_{66}$ : the correlation matrix is $(1 / 20) Z^{(t) T}{ }_{20,6} Z^{(t)}{ }_{20,6}=R_{6,6}$. The matrix $C_{66}$ is known, the matrix $\Lambda_{66}$-restored, the matrix $R_{6,6}$ is unknown. Mathematical problems are solved: optimization, inverse spectral; multidimensional statistical problems - Inverse Problem of Principal Component Analysis. Result - Table 4 demonstrates the realism of the values of 6 property values of grain crops.

Key words: parameters, variables for the matrix of real measured data, realistic numbers from the $\Lambda$ sample.

Language: Russian

Citation: Zhanatauov, S. U., \& Agaev, A. (2019). A giving realism to the property values of the cereal crops in the model $\lambda$-sample. ISJ Theoretical \& Applied Science, 05 (73), 501-509.

Soi: http://s-o-i.org/1.1/TAS-05-73-76 Doi: crossef https://dx.doi.org/10.15863/TAS.2019.05.73.76

\section{ПРИДАНИЕ РЕАЛИСТИЧНОСТИ ЗНАЧЕНИЯМ ПРИЗНАКОВ ЗЕРНОВОЙ КУЛЬТУРЫ В МОДЕЛЬНОЙ $\Lambda$-ВЫБОРКЕ}

Аннотация: В статье дано описание применения ОМ ГК: $\left(\boldsymbol{C}_{66,}, \Lambda_{66}\right)=>\left(R_{6,6}, U^{(t)}{ }_{20,6}, Y^{(t)}{ }_{20,6}=U^{(\ell t)}{ }_{20,6} \Lambda^{1 / 2}{ }_{66}\right.$, $\left.I_{6,6}=Z^{(t)}{ }_{20,6}=Y^{(t)}{ }_{20,6} C_{6,6}^{T}\right), t=1, \ldots, k_{t}<\infty$. Выгборка $U_{20,6} \quad$ и-переменных имеет корреляционную матрицу $(1 / 20) U^{(t) T} 20,6 U^{(t)} 20,6=\operatorname{diag}(1, \ldots, 1), \quad$ выборка $\quad$-переменных $Y_{20,6}=U_{20,6} \Lambda^{1 / 2}{ }_{66} \quad$ - ковариационную матрицу $\Lambda_{n n}=\operatorname{diag}\left(\lambda_{1}, \lambda_{2}, \ldots, \lambda_{6}\right), \quad$ выборка $\quad z$-переменных $Z^{(t)}{ }_{20,6}=Y^{(t)}{ }_{20,6} C_{6,6}^{T}=U^{(t)} 20,6 \Lambda_{66}^{1 / 2} \quad$ - корреляциионную матрииу $(1 / 20) Z_{20,6}^{(t) T} Z_{20,6}^{(t)}=R_{6,6}$. Матрица $C_{66}$, известна, матрица $\Lambda_{66}$-восстановлена, матрица $R_{6,6}-$ неизвестна. Решаются математические задачи: оптимизачионная, обратная спектральная; многомерные статистические задачи -Обратная Задача Анализа Главных Компонент. Результат- Таблица 4 демонстрирует реалистичности значениям 6 признаков зерновой культуры.

Ключевые слова: параметры переменные для матрицы реальных измеренных данных, реалистичность чисел из $\Lambda$-выборки.

\section{Введение}

В разных сферах могут применяться различные процедуры моделирования цифровых данных, где на конечном этапе необходимо визуализировать реалистичность модельных чисел, имеющих размерность. Расчетное число $\$ 300,78336$ должно быть округлено до двух знаков после запятой. При этом, если каждое 


\begin{tabular}{|c|c|c|c|c|c|c|}
\hline \multirow{4}{*}{ Impact Factor: } & ISRA (India) & $=3.117$ & SIS (USA) & $=0.912$ & ICV (Poland) & $=6.630$ \\
\hline & ISI (Dubai, UAI & $=0.829$ & РИНЦ (Russia & $=0.156$ & PIF (India) & $=1.940$ \\
\hline & GIF (Australia) & $=0.564$ & ESJI (KZ) & $=8.716$ & IBI (India) & $=4.260$ \\
\hline & JIF & $=1.500$ & SJIF (Morocco & $=5.667$ & OAJI (USA) & $=0.350$ \\
\hline
\end{tabular}

слагаемое нескольких слагаемых нужно округлить, то их сумма должна совпасть с суммарным округленным числом. Сумма слагаемых (в денежных единицах) по горизонтальной строке должна равняться сумме слагаемых (в денежных единицах) по вертикальному столбцу.

Некоторые слагаемые являются расчетными, a не «кассовыми», полученными наличными денежными знаками с точностью до 1 цента. Недопустимо присутствие 78,336 цента в качестве слагаемого. Если даже оно получено в качестве итогового значения фондового рынка, валютных обменных операций. Существуют разные формы округления (математическое, банковское, случайное и др.) и разные ситуации их применения при моделировании реальных процессов. Во всех этих формах округления "лишние" цифровые знаки обнуляют, а предшествующий им знак корректируется по какому-либо правилу.

В моделировании значений признаков, измеряемых в весьма разных единицах измерения и их масштабах существует прием «стандартизация». в омгк, применяемым нами, для моделирования реалистичных значений 6 признаков зерновой культуры, моделируются многомерная $\Lambda$-выборка является многомерной стандартизованной выборкой.

Рассмотрим основные моменты придания реалистичности числовым значениям 6 признаков зерновой культуры, получаемых в результате применения сложных математических моделей многомерных объектов в виде векторов, в виде матриц, имеющих разные свойства, но по-своему отражающие информацию, знания, скрытые в них.

Значения функции $\sin$ материализуются, если иметь точки с координатами $(\mathrm{y}, \mathrm{z})$ удовлетворяют формуле $\mathrm{z}=\mathrm{kx} \sin (\mathrm{y} / \mathrm{a})$ и требуемым формам округления. Здесь цель: отказ от прямых линий и углов (в архитектуре) в пользу более естественных, «природных» линий. Это достигается моделированием по модельным линиям из точек (y,z), удовлетворяющих формуле $\mathrm{z}=\mathrm{kx} \sin (\mathrm{y} / \mathrm{a})$ и материализуются в виде поверхности Гауди.

Мы ниже применяем символы $\mathrm{z}, \mathrm{k}, \mathrm{x}, \sin , \mathrm{y}, \mathrm{a}$, но наши модельные точки не материализуются в поверхности, но используются в ІТ-технологии для достижения конкретной, измеримой, достижимой, актуальной, ограниченной сроком цели, сформулированной в заглавии статьи.

Наши цифровые модельные данные для этих значений признаков зерновой культуры (в модельной $\Lambda$-выборке) должны, как требуется стандартами, отображены в виде таблицы с соблюдением 6 форм округления (Таблица 4).

В расчетах по моделированию задача округления наших модельных данных является удаленной конечной целью. После нее следует расчет показателя «урожайность с одного гектара пахотной земли». Этот показатель является наиважнейшим, мы стремились выявить регулируемые параметры (f-,BDE-E- параметры, матрицы $\Lambda_{\mathrm{nn}}, \mathrm{C}_{\mathrm{nn}}$ ), переменные (a-,b-,z-,u-,y-), учет которых позволил достичь высокой степени неопределенности у значений 6 z-переменных в формуле $\mathrm{x}_{\mathrm{ij}}{ }^{\circ}=\mathrm{Z}_{\mathrm{ij}} \mathrm{S}_{\mathrm{j}}+\mathrm{x}_{\mathrm{j}}{ }^{\mathrm{cp}}$, где доминирующий вклад в сумму вносит слагаемое $\mathrm{x}_{\mathrm{j}}^{\mathrm{cp}}$, а второе слагаемое $\mathrm{Z}_{\mathrm{ij}} \mathrm{S}_{\mathrm{j}}$, есть случайное число $\mathrm{z}_{\mathrm{ij}}$ с постоянным множителем $\mathrm{s}_{\mathrm{j}}$. Возможен отбор смоделированных матриц значений 6 z-переменных по разным критериям, налагаемым на приведенные выше параметры и переменные. Если значения чисел $\mathrm{x}_{\mathrm{j}}^{\mathrm{cp}}$ и $\mathrm{Z}_{\mathrm{ij}} \mathrm{s}_{\mathrm{j}}$, удовлетворяют форме округления, то значение числа может не удовлетворять той же форме округления. В нашем случае из видимых цифровых знаков в числах 6 средних видно, что они не удовлетворяют формам округления ни одно из двух слагаемых. Формы округления важны для практических работников агробизнеса.

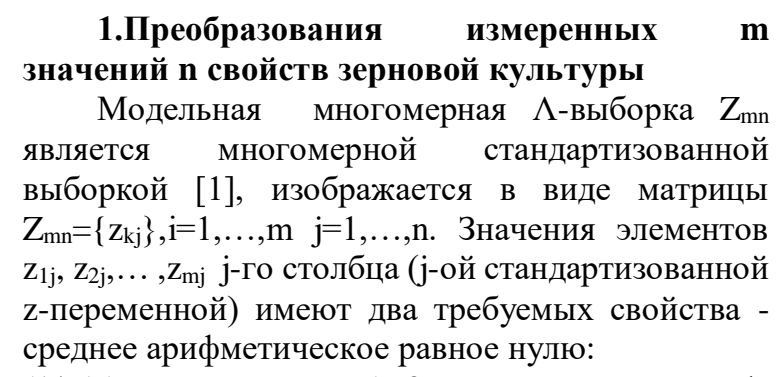
$(1 / \mathrm{m})\left(\mathrm{z}_{1 \mathrm{j}},+\mathrm{z}_{2 \mathrm{j}}+\ldots+\mathrm{z}_{\mathrm{mj}}\right)=0$, дисперсия равна 1 : $(1 / \mathrm{m})\left({ }^{\mathrm{z} 2}{ }_{1 \mathrm{j}}+\mathrm{z}^{2}{ }_{2 \mathrm{j}}+\ldots+\mathrm{z}^{2}{ }_{\mathrm{mj}}\right)=1$, сумма дисперсий $\mathrm{z}-$ переменных равна $\mathrm{n}=6$. Матрица $\mathrm{Z}_{\mathrm{mn}}$ интерпретируется во-первых, как многомерная выборка. В когнитивных моделях извлечения знаний из цифровых данных [2-6] значения $\mathrm{Z}_{\mathrm{ik}}$, $\mathrm{i}=1, \ldots, \mathrm{m}, \mathrm{k}=1, \ldots, \mathrm{n}, \mathrm{z}$-переменных из $\mathrm{n}$-мерной выборки $Z_{\mathrm{mn}}$ выступают в другой принципиально отличающейся от прежней роли интерпретируются (рассматриваются при когнитивном моделировании) как значения $\mathrm{Z}_{\mathrm{ki}}$ и $\mathrm{Z}_{\mathrm{kj}}$ пропорциональных изменчивостей пар переменных $\mathrm{r}_{\mathrm{ij}}=\operatorname{corr}\left(\mathrm{z}_{\mathrm{i}}, \mathrm{z}_{\mathrm{j}}\right): \quad \mathrm{z}_{\mathrm{ki}}=\mathrm{r}_{\mathrm{ij}} \mathrm{z}_{\mathrm{kj}}$. Bce пары значений пропорциональных изменчивостей сосредоточены в матрице $\mathrm{Z}_{\mathrm{mn}}$. Преобразования при стандартизации значений $\mathrm{x}_{\mathrm{ij}}^{0}$ превратились в значения $\mathrm{Z}_{\mathrm{ij}}$ изменчивостей (присущих значению $\mathrm{x}_{\mathrm{ij}}{ }_{\text {ij }}$. Этим мы фиксируем наличие и величину изменчивости в матрице $Z_{\mathrm{mn}}$, рассматриваемой как единый объект, как единое целое. Если у значения $\mathrm{x}_{\mathrm{ij}}^{0}$ отсутствует изменчивость, то имеем $\mathrm{z}_{\mathrm{ij}}=0, \mathrm{x}_{\mathrm{ij}}=\mathrm{x}^{\mathrm{cp}}{ }_{\mathrm{j}}$. Наличие ненулевой изменчивости у элементов $\left\{\mathrm{z}_{\mathrm{ij}}\right\}$ очень важно. Это дает наличие достаточно заметных по величине значений компонентов собственных векторов индикаторов присутствия когнитивных знаний [26]. А Наличие ненулевой изменчивости у 


\begin{tabular}{|c|c|c|c|c|c|c|}
\hline \multirow{4}{*}{ Impact Factor: } & ISRA (India) & $=3.117$ & SIS (USA) & $=0.912$ & ICV (Poland) & $=6.630$ \\
\hline & ISI (Dubai, UAE & $=0.829$ & РИНЦ (Russia & $=0.156$ & PIF (India) & $=1.940$ \\
\hline & GIF (Australia) & $=0.564$ & ESJI (KZ) & $=8.716$ & IBI (India) & $=4.260$ \\
\hline & JIF & $=1.500$ & SJIF (Morocce & $=5.667$ & OAJI (USA) & $=0.350$ \\
\hline
\end{tabular}

элементов $\left\{\mathrm{z}_{\mathrm{ij}}\right\}$ дает близкие к 1 значения коэффициента пропорциональности $\mathrm{r}_{\mathrm{ij}}$ такого, что $\mathrm{Z}_{\mathrm{ki}}=\mathrm{r}_{\mathrm{ij}} \mathrm{Z}_{\mathrm{kj}}$. Наличие высоких коэффициентов корреляций дает спектр $\Lambda_{\mathrm{nn}}=\operatorname{diag}\left(\lambda_{1}, \lambda_{2}, \ldots, \lambda_{\mathrm{n}}\right)$, элементы которого на графике изображают круто наклоненную кривую, что она задает понятную для аналитика свою связь между значениями $6 \mathrm{f}-$ параметров спектра $\Lambda_{\mathrm{nn}}=\operatorname{diag}\left(\lambda_{1}, \lambda_{2}, \ldots, \lambda_{\mathrm{n}}\right)$. После стандартизации начинаются более важные преобразования для полученной матрицы $\mathrm{Z}_{\mathrm{mn}}$.

Термины «стандартизованное» значение и «пропорциональные изменчивости» $\mathrm{Z}_{\mathrm{ki}}$ и $\mathrm{Z}_{\mathrm{kj}}$ разнятся своими возможностями - в первом случае имеются в виду преобразованные безразмерные значения, полученные от исходной реальной выборки данных $\mathrm{X}_{20,6}^{0}$, рассматриваемой как отдельный объект, выбранный из генеральной совокупности с неизвестным законом распределения. То есть $\mathrm{n}-$ мерная выборка $Z_{\mathrm{mn}}$ содержит, во-первых, преобразованные данные [1]. Значения этих данных, если их рассматривать в рамках когнитивной модели извлечения знаний из данных [2-6], являются (интерпретируются) иначе (имеют другой содержательный смысл): значение $\mathrm{Z}_{\mathrm{ki}}$ равно изменчивости k-го значения i-oй $\mathrm{z}$ переменной [7]. Во-вторых, $\mathrm{n}$-мерная выборка $\mathrm{Z}_{\mathrm{mn}}$ содержит $\mathrm{m}$ значений $\mathrm{Z}_{\mathrm{kj}}$ изменчивостей каждой из n z-переменных. Нами установленное соответствие [7]:

преобразованные данные (в модели ПМ ГК+ОМ ГК) $\Rightarrow$ значения изменчивостей каждой из n z-переменных (в когнитивной модели извлечения знаний из данных $Z_{\mathrm{mn}}$ ).

Значение $Z_{k i}$, равное изменчивости k-го значения i-ой $\mathrm{z}$-переменной., является случайным множителем при значении $\mathrm{c}_{\mathrm{ij}} \mathrm{j}$-го «веса» $\mathrm{z}_{\mathrm{ki}} \mathrm{c}_{\mathrm{ij}}$.Это слагаемое $-\mathrm{Z}_{\mathrm{ki}} \mathrm{c}_{\mathrm{ij}}$, является частью суммы слагаемых $\mathrm{y}_{\mathrm{kj}}=\mathrm{z}_{\mathrm{k} 1} \mathrm{c}_{1 \mathrm{j}}+\mathrm{Z}_{\mathrm{k} 2} \mathrm{c}_{2 \mathrm{j}}+\ldots+\mathrm{z}_{\mathrm{kjn}} \mathrm{c}_{\mathrm{nj}}$, $\mathrm{i} \in\{1, \ldots, \mathrm{n}\}$.

Указанная сумма слагаемых является новой y-переменной. Так как $\mathrm{j}=1, \ldots, n$, то число $\mathrm{y}$ переменных равно $\mathrm{n}$. Значения у-переменных образуют матрицу $\mathrm{Y}_{\mathrm{mn}}$, равную произведению матрицы z-переменных на матрицу $\mathrm{C}_{\mathrm{nn}}$ собственных векторов (значений «весов»): $\mathrm{Y}_{\mathrm{mn}}=\mathrm{Z}_{\mathrm{mn}} \mathrm{C}_{\mathrm{nn}}$.

Здесь одна у-переменная равна линейной комбинации $\mathrm{n}$ z-переменных: $\mathrm{y}_{\mathrm{ij}}=\mathrm{z}_{\mathrm{i} 1} \mathrm{c}_{1 \mathrm{j}}+\mathrm{z}_{\mathrm{i} 2} \mathrm{c}_{2 \mathrm{j}}+\ldots+\mathrm{z}_{\mathrm{in}} \mathrm{c}_{\mathrm{nj}}$, у-переменная не являются стандартизованной, следовательно, имеют разные значения дисперсий $\lambda_{1}, \lambda_{2}, \ldots, \lambda_{\mathrm{n}}$, их совокупность удовлетворяет

$(1 / \mathrm{m}) \mathrm{Y}^{\mathrm{T}}{ }_{\mathrm{mn}} \mathrm{Y}_{\mathrm{mn}}=\Lambda_{\mathrm{nn}}=\operatorname{diag}\left(\lambda_{1}, \lambda_{2}, \ldots, \lambda_{\mathrm{n}}\right)$. формуле дисперсии не стандартизованных у-переменных зависят от корреляционной матрицы $\mathrm{R}_{\mathrm{nn}} \mathrm{n}$ стандартизованных
$(1 / \mathrm{m}) \mathrm{Z}^{\mathrm{T}}{ }_{\mathrm{mn}} \mathrm{Z}_{\mathrm{mn}}=\mathrm{R}_{\mathrm{nn}}$. Матрица корреляций нам не известна. Если бы она была известна, то нашей работы не было бы. Для известной корреляционной матрицы $\mathrm{R}_{\mathrm{nn}}$, являющейся симметрической матрицей всегда существует пара матриц $\left(\Lambda_{\mathrm{nn}}, \mathrm{C}_{\mathrm{nn}}\right)$, где $\Lambda_{\mathrm{nn}}-$ называется матрицей собственных чисел, $\mathrm{C}_{\mathrm{nn}}$ - матрица собственных векторов. Между этими переменными имеется корреляционная связь: $\operatorname{corr}\left(\mathrm{z}_{\mathrm{i}}, \mathrm{y}_{\mathrm{j}}\right)=\mathrm{c}_{\mathrm{ij}}$, элементы $(\mathrm{y}, \mathrm{z})$-корреляций образуют несимметрическую матрицу $\mathrm{C}_{\mathrm{nn}}=(1 / \mathrm{m}) \mathrm{Z}^{\mathrm{T}}{ }_{\mathrm{mn}}\left[\mathrm{Y}_{\mathrm{mn}} \Lambda^{(-1)}\right], \quad$ являющуюся матрицей линейного преобразования $\mathrm{n} \mathrm{Z}$ переменных в $\mathrm{n}$ у -переменных: матрица $\mathrm{Z}_{\mathrm{mn}}$ преобразуем в матрицу $\mathrm{Y}_{\mathrm{mn}}=\mathrm{Z}_{\mathrm{mn}} \mathrm{C}_{\mathrm{nn}}$.

Таким образом имеем ПМ ГК [8]: $\mathrm{Z}_{\mathrm{mn}}=>\left(\mathrm{R}_{\mathrm{nn}}, \mathrm{C}_{\mathrm{nn}}, \Lambda_{\mathrm{nn}}, \mathrm{Y}_{\mathrm{mn}}\right)$.

Для фактических $20 \times \times 6=120$ значений известны следующие параметры, не все значения из них соответствуют форме округления.

1. Каждый элемент $\mathrm{Z}_{\mathrm{ij}}$ из матрицы $\mathrm{Z}_{\mathrm{mn}}$ получен преобразованием элемента $\mathrm{x}_{\mathrm{ij}}{ }^{0}$ в безразмерный элемент матрицы $\mathrm{Z}_{131,6}=\left\{\mathrm{Z}_{\mathrm{ij}}\right\}$, $\mathrm{i}=1, \ldots, 20, \mathrm{j}=1, \ldots, 6$, где $\mathrm{z}$-переменные имеют значения $\quad \mathrm{z}_{\mathrm{ij}}=\left(\mathrm{x}_{\mathrm{ij}}{ }^{\circ}-\mathrm{x}_{\mathrm{j}}{ }^{\mathrm{cp}}\right) / \mathrm{s}_{\mathrm{j}}, \mathrm{x}_{\mathrm{ij}}=\mathrm{x}_{\mathrm{ij}}{ }^{\circ}-\mathrm{x}_{\mathrm{j}}^{\mathrm{cp}}, \quad \mathrm{i}=1, \ldots, 20$, $\mathrm{j}=1, \ldots, 6$, а значения компонент векторов являются постоянными: $\mathrm{x}_{\mathrm{j}}^{\mathrm{c}}=\left(\mathrm{x}_{1, \mathrm{j}}^{\circ}+\ldots+\mathrm{x}_{20, \mathrm{j}}^{\circ}\right) / 20$, $\mathrm{s}_{\mathrm{j}}{ }^{2}=\left(\mathrm{x}_{1 \mathrm{j}}{ }^{2}+\ldots+\mathrm{x}_{20, \mathrm{j}}{ }^{2}\right) / 20$. Вектор средних [11] $\mathrm{x}^{\mathrm{cp}}{ }_{16}=(45,75,106,1,19,15,1,1367,40,45,27,65)$ и вектор выборочных стандартных отклонений [11] $\mathrm{s}_{16}=\operatorname{diag}(4,4931,7,5425,1,711,0,1532,5,0742,2,7798)$

2. Матричные равенства ПМГК: $(1 / m) Z^{T}{ }_{m n} Z_{m n}=R_{n n}, R_{n n} C_{n n}=C_{n n} \Lambda_{n n}$,

$\mathrm{C}^{\mathrm{T}}{ }_{\mathrm{nn}} \mathrm{C}_{\mathrm{nn}}=\mathrm{C}_{\mathrm{nn}} \mathrm{C}^{\mathrm{T}}{ }_{\mathrm{nn}}=\mathrm{I}_{\mathrm{nn}}, \quad \operatorname{diag}\left(\mathrm{R}_{\mathrm{nn}}\right)=(1, \ldots, 1)$, $\operatorname{tr}\left(R_{\mathrm{nn}}\right)=1+1+\ldots+1=\operatorname{tr}\left(\Lambda_{\mathrm{nn}}\right)=\lambda_{1}+\ldots+\lambda_{\mathrm{n}}=\mathrm{n}$,

$\lambda_{1} \geq \ldots \geq \lambda_{\mathrm{n}} \geq 0[1,37], \mathrm{Y}_{\mathrm{mn}}=\mathrm{Z}_{\mathrm{mn}} \mathrm{C}_{\mathrm{nn}}, \quad(1 / \mathrm{m}) \mathrm{Y}^{\mathrm{T}}{ }_{\mathrm{mn}} \mathrm{Y}_{\mathrm{mn}}=\Lambda_{\mathrm{nn}}$, $\mathrm{C}_{\mathrm{nn}}=(1 / \mathrm{m}) \mathrm{Z}_{\mathrm{mn}}^{\mathrm{T}}\left[\mathrm{Y}_{\mathrm{mn}} \Lambda^{(-1)}\right]$.

3. Функциональные ограничения для значений элементов спектра $\Lambda_{\mathrm{nn}}=\operatorname{diag}\left(\lambda_{1}, \lambda_{2}, \ldots, \lambda_{\mathrm{n}}\right): \mathrm{f}_{1}\left(\Lambda_{\mathrm{nn}}\right)=\quad \lambda_{1}+\ldots$ $\left.+\lambda_{\mathrm{n}}\right)=\mathrm{n}, \mathrm{f}_{2}\left(\Lambda_{\mathrm{nn}}\right)=\left(\lambda^{2}{ }_{1}+\ldots+\lambda^{2}{ }_{\mathrm{n}}\right), \mathrm{f}_{3}\left(\Lambda_{\mathrm{nn}}\right)=\lambda_{1} / \lambda_{\mathrm{n}}, \mathrm{f}_{4}\left(\Lambda_{\mathrm{nn}}\right)=$ $\left(\lambda_{1}+\ldots+\lambda_{\ell}\right) / \mathrm{n}<1 . \mathrm{f}_{5}\left(\Lambda_{\mathrm{nn}}\right)=\lambda_{1} \times \lambda_{2} \times \lambda_{3} \times \ldots \times \lambda_{\mathrm{n}}, \mathrm{f}_{6}\left(\Lambda_{\mathrm{nn}}\right)=\lambda_{1} / \lambda$ $\left.{ }_{2}+\ldots+\lambda_{\mathrm{n}-1} / \lambda_{\mathrm{n}}\right)$. Их значения равны $[11]: \varphi=0,3839$, $\mathrm{f}_{1}=6, \quad \mathrm{f}_{2}=10,56, \mathrm{f}_{3}=627,9, \ell=3, \mathrm{f}_{4}=0,8806, \mathrm{f}_{5}=1,7 \mathrm{E}-3$, $\mathrm{f}_{6}=260,17, \lambda_{1}=2.5117, \lambda_{2}=1.592, \lambda_{3}=1.18$. Здесь $\mathrm{f}_{2}\left(\Lambda_{66}\right)=\left(\lambda^{2}{ }_{1}+\ldots+\lambda^{2}{ }_{6}\right)=\operatorname{tr}\left(\mathrm{R}_{66}^{\mathrm{T}} \mathrm{R}_{66}\right)=10,56$.

4.1. Если известны b-параметры $\left(\lambda_{\mathrm{i}}=\mathrm{b}_{\mathrm{i}} \lambda_{\mathrm{i}-1}\right)$ спектра $\Lambda_{\mathrm{nn}}=\operatorname{diag}\left(\lambda_{1}, \lambda_{2}, \ldots, \lambda_{\mathrm{n}}\right), \quad$ то определены элементы спектра по следующим формулам: $\lambda_{1}=\mathrm{n} /$ $\left(1+b_{2} \times b_{3}+b_{2} \times b_{3} \times b_{4}+\ldots+b_{2} \times \ldots \times b_{n}\right), b_{i}=\lambda_{i} / \lambda_{\mathrm{i}-1} \leq 1 .$,

$\lambda_{\mathrm{j}}=\left(\mathrm{b}_{2} \times \ldots \times \mathrm{b}_{\mathrm{j}}\right) \times \lambda_{1}, \quad \mathrm{i}=2, \ldots, \mathrm{n}, \quad$ где сохраняются свойства элементов спектра корреляционной матрицы: $\lambda_{1}+\ldots+\lambda=\mathrm{n}, \lambda_{1} \geq \ldots \geq \lambda_{\mathrm{n}} \geq 0$,

4.2. Если известны а-параметры $\left(\lambda_{\mathrm{i}-1}=\mathrm{a}_{\mathrm{i}} \lambda_{\mathrm{i}}\right)$ спектра $\Lambda_{\mathrm{nn}}=\operatorname{diag}\left(\lambda_{1}, \lambda_{2}, \ldots, \lambda_{\mathrm{n}}\right), \quad$ то определены элементы спектра по следующим формулам: $\lambda_{\mathrm{n}}=\mathrm{n} /$ $\left(1+a_{2} \times \ldots \times a_{n}+\quad \ldots+a_{n-1} \times a_{n}\right), \lambda_{j}=\left(a_{i+1} \quad \times \ldots \times a_{n}\right) \times \lambda_{n}$, $\mathrm{i}=2, \ldots, \mathrm{n}-1$, где сохраняются свойства элементов 


\begin{tabular}{|c|c|c|c|c|c|c|}
\hline \multirow{4}{*}{ Impact Factor: } & ISRA (India) & $=3.117$ & SIS (USA) & $=0.912$ & ICV (Poland) & $=6.630$ \\
\hline & ISI (Dubai, UAE & $=0.829$ & РИНЦ (Russia & $=0.156$ & PIF (India) & $=1.940$ \\
\hline & GIF (Australia) & $=0.564$ & ESJI (KZ) & $=8.716$ & IBI (India) & $=4.260$ \\
\hline & JIF & $=1.500$ & SJIF (Morocce & $=5.667$ & OAJI (USA) & $=0.350$ \\
\hline
\end{tabular}

спектра корреляционной матрицы: $\lambda_{1}+\ldots+\lambda=\mathrm{n}$, $\lambda_{1} \geq \ldots \geq \lambda_{n} \geq 0$.

Мы зафиксировали заданные значения [11] входных параметров (для достижения адекватности реальной многомерной выборке) для моделируемой ниже выборки. В Таблице 1 приведены параметры восстановленного спектра $\Lambda_{66}$, вычисленные с высокой степенью точности. Чего не требуется при визуализации значений таблицы $\mathrm{X}^{0} 20,6$.

Таблица 1. «Динамические» параметры спектра

\begin{tabular}{|l|l|l|l|l|l|l|}
\hline $\mathrm{j}$ & 1 & 2 & 3 & 4 & 5 & 6 \\
\hline$\lambda_{\mathrm{j}}$ & 2,5117 & 1,592 & 1,1800 & 0,462221049 & 0,173723201 & 0,08035575 \\
\hline$\Delta_{\mathrm{j}}=\lambda_{\mathrm{j}} / 6$ & 0,4186 & 0,2653 & 0,1967 & 0,0770 & 0,0290 & 0,0134 \\
\hline $\mathrm{D}(\mathrm{j})=\left(\lambda_{1}+\ldots+\lambda_{\mathrm{j}}\right) / 6$ & 0,4186 & 0,6840 & 0,8806 & 0,9577 & 0,9866 & 1,0000 \\
\hline $\mathrm{a}_{\mathrm{j}}=\lambda_{\mathrm{j}} / \lambda_{\mathrm{j}-1}$ & & 1,577701 & 1,34915 & 2,552891096 & 2,660675403 & 2,161926207 \\
\hline
\end{tabular}

Таблица 2. Матрица собственных векторов $\mathrm{C}_{66}$

\begin{tabular}{|l|l|l|l|l|l|l|}
\hline ROW 1 & 0,5106 & $-0,3477$ & $-0,6143$ & $-0,3411$ & 0,2672 & $-0,2307$ \\
\hline ROW 2 & 0,0665 & $-0,4642$ & $-0,0354$ & 0,3835 & $-0,6793$ & $-0,4128$ \\
\hline ROW 3 & 0,4569 & $-0,3185$ & 0,0972 & 0,4628 & 0,109 & 0,674 \\
\hline ROW 4 & 0,2923 & $-0,6128$ & 0,2572 & $-0,5981$ & $-0,1469$ & 0,306 \\
\hline ROW 5 & 0,5129 & 0,421 & 0,072 & $-0,3978$ & $-0,5934$ & 0,21 \\
\hline ROW 6 & 0,4215 & $-0,0976$ & 0,7352 & $-0,0796$ & 0,2856 & $-0,4294$ \\
\hline
\end{tabular}

Здесь $Z_{20,6}$-входной элемент решенной ранее ПЗ АГК [1,8]: $\mathrm{Z}_{131,6}=>\left(\mathrm{R}_{66}, \quad \mathrm{C}_{66}, \Lambda_{66}, \mathrm{Y}_{131,6}\right)$, а использованная нами известная матрица собственных векторов $\mathrm{C}_{66}$ (Таблица 2) вычислена при решении прямой спектральной задачи (ПСЗ) диагонализации симметрической матрицы [1] $\mathrm{R}_{\mathrm{nn}}=>\left(\mathrm{C}_{\mathrm{nn}}, \Lambda_{\mathrm{nn}}\right)$. Матрица $\mathrm{R}_{\mathrm{nn}}$ потеряна, $\Lambda_{\mathrm{nn}}-$ была восстановлена $[9,11]$ по неполной матрице, где отсутствовали не доминирующие элементы $\lambda_{4}, \lambda_{5}, \lambda_{6}$.

Вычисленные по алгоритму (восстановленные) значения оценок не доминирующих элементов спектра равны: $\lambda_{4}=0.307010656, \lambda_{5}=0.018512809, \lambda_{6}=0.08035575$. Теперь мы имеем полный спектр $\Lambda_{66}=\operatorname{diag}(2.5117,1.592,1.18, \quad 0.462221049$, $0.173723201,0.08035575)$.

Считаем известными 3 домин-х элемента $\Lambda_{66}=\operatorname{diag}(2.5117,1.592,1.18$. Полагаем известными и правильно оцененными значения -параметров $\mathrm{f}_{1}$ $\mathrm{f}_{2}, \mathrm{f}_{3}, \mathrm{f}_{4}$. Равенство этих значений 4-х функций числовым значениям 60,7476, 31,257, 0,8806, вводим в модель как ограничения на элементы $\lambda_{4}$, $\lambda_{5}, \lambda_{6}$.

2.Модельная многомерная выборка $Z_{20,6}$ (с модельными значениями 6 z-переменных)

Многомерная $\Lambda$-выборка $Z_{\mathrm{mn}}$, как ассоциированное решение ОЗ АГК [1], является многомерной стандартизованной выборкой, изображается в виде матрицы $\mathrm{Z}_{\mathrm{mn}}=\left\{\mathrm{z}_{\mathrm{kj}}\right\}, \mathrm{i}=1, \ldots, \mathrm{m}$ $\mathrm{j}=1, \ldots, n$. Значения элементов $\mathrm{z}_{1 \mathrm{j}}, \mathrm{z}_{2 \mathrm{j}}, \ldots, \mathrm{z}_{\mathrm{mj}} \mathrm{j}$-го столбца (j-ой стандартизованной $\mathrm{Z}$-переменной) имеют два требуемых свойства - среднее арифметическое равное нулю: $(1 / \mathrm{m})\left(\mathrm{z}_{1 \mathrm{j}},+\mathrm{z}_{2 \mathrm{j}}+\ldots\right.$ $\left.+\mathrm{z}_{\mathrm{mj}}\right)=0$,дисперсия равна $1:(1 / \mathrm{m})\left({ }^{\mathrm{z} 2}{ }_{1 \mathrm{j}}+\mathrm{z}^{2}{ }_{2 \mathrm{j}}+\ldots+\right.$ $\left.\mathrm{z}^{2} \underset{\mathrm{mj}}{ }\right)=1$, сумма дисперсий $\mathrm{z}$-переменных равна $\mathrm{n}=6$. Матрица $\mathrm{Z}_{\mathrm{mn}}$ интерпретируется во-первых, как многомерная выборка, во-вторых - как матрица значений изменчивостей $\mathrm{n}$ z-переменных. Eе элемент - результат двух преобразований значения $\mathrm{x}_{\mathrm{ij}}^{0}$ исходного натурального измерения прибором. Так как коэффициент корреляции Пирсона показывает (через свое значение $\mathrm{r}_{12}$ ) насколько выражена пропорциональная изменчивость двух переменных $\mathrm{z}_{1}$ и $\mathrm{z}_{2}: \mathrm{z}_{\mathrm{k} 1}=\mathrm{r}_{12} \mathrm{Z}_{\mathrm{k} 2}$, $1 \leq \mathrm{r}_{12} \leq 1$, при всех $\mathrm{k}=1, \ldots, \mathrm{m}$, то $\mathrm{Z}_{\mathrm{mn}}$ является матрицей [7]изменчивостей.

Мы имеем матрицу значений $\mathrm{x}_{\mathrm{ij}}$ исходных натуральных измерений, но для вычисления корреляционной матрицы $\mathrm{R}_{\mathrm{nn}}$ мы должны иметь матрицу изменчивостей для матрицы исходных данных. Матрица исходных данных характеризуется своей матрицей изменчивостей $\mathrm{Z}_{\mathrm{mn}}$ и векторами [9]: средних $\mathrm{x}^{\mathrm{cp}}{ }_{16}=(45,75,106,1$, $19,15,1,1367,40,45, \quad 27,65)$ и стандартных отклонений $\mathrm{s}_{16}=\operatorname{diag}(4,4931,7,5425,1,711,0,1532,5,0742,2,7798)$ 


\begin{tabular}{|c|c|c|c|c|c|c|}
\hline \multirow{4}{*}{ Impact Factor: } & ISRA (India) & $=3.117$ & SIS (USA) & $=0.912$ & ICV (Poland) & $=6.630$ \\
\hline & ISI (Dubai, UAE & $=0.829$ & РИНЦ (Russia & $=0.156$ & PIF (India) & $=1.940$ \\
\hline & GIF (Australia) & $=0.564$ & ESJI (KZ) & $=8.716$ & IBI (India) & $=4.260$ \\
\hline & JIF & $=1.500$ & SJIF (Morocce & $=5.667$ & OAJI (USA) & $=0.350$ \\
\hline
\end{tabular}

Такая замена одного объекта тремя другими объектами позволяет применять вместо недешевых n приборов другие моделируемые матрицы: Z,R, C, $\Lambda, \mathrm{Y}$.

Матрицы Z,R, C, $\Lambda$, Y разделяются в ПМ ГК и В ОМ ГК на 2 группы: входные и выходные. Одна из задач - ПЗ АГК решается в модели ОМ ГК: $\mathrm{Z} \Rightarrow(\mathrm{R}, \mathrm{C}, \Lambda, \mathrm{Y})$, где входным объектом является матрица изменчивостей $Z_{\mathrm{mn}}$. Векторы $\mathrm{x}^{\mathrm{cp}}{ }_{16}$ и $\mathrm{s}_{16}$ пока не участвуют в шагах достижения нашей цели. Шаг (длина единичного шага) коэффициента изменчивости $\mathrm{s}_{\mathrm{j}}$ и величина изменчивости $\mathrm{Z}_{\mathrm{ij}} \mathrm{j}$-ой $\mathrm{z}$-переменной определяют изменяемую часть $\mathrm{sj}_{\mathrm{j}} \mathrm{ij}$ значения исходной измеренной $\mathrm{x}_{\mathrm{ij}}^{0}=\mathrm{s}_{\mathrm{j}} \mathrm{z}_{\mathrm{ij}}+\mathrm{x}^{\mathrm{cp}} \mathrm{j}_{\mathrm{j}}$, где постоянная часть пока не участвует в наших вычислениях.

Матрица $Z_{m n}$ и определяет матрицу $R_{n n}$ : $(1 / \mathrm{m}) \mathrm{Z}_{\mathrm{mn}}^{\mathrm{T}} \mathrm{Z}_{\mathrm{mn}}=\mathrm{R}_{\mathrm{nn}}$. Значение элемента т матрицы $\mathrm{R}_{\mathrm{nn}} \mathrm{r}_{12}$ таково, что вектор-столбец $\mathbf{z}_{1}=\left(\mathrm{z}_{11}, \ldots, \mathrm{Z}_{1 \mathrm{~m}}\right)^{\mathrm{T}}$, значений переменной $\mathrm{Z}_{1}$ равно вектор-столбцу $\left(\mathrm{r}_{12} \mathrm{Z}_{\mathrm{i} 2}, \ldots, \mathrm{r}_{12} \mathrm{Z}_{\mathrm{m} 2}\right)^{\mathrm{T}}$ значений переменной $\left(\mathrm{r}_{12}\right) \mathbf{z} 2$. Коэффициенты $(\mathrm{z}, \mathrm{z})$-корреляции $\mathrm{r}_{\mathrm{ij}}=\operatorname{corr}\left(\mathrm{z}_{\mathrm{i}}, \mathrm{z}_{\mathrm{j}}\right)$ образуют симметрическую матрицу $\mathrm{R}_{\mathrm{nn}}=$ $(1 / \mathrm{m}) \mathrm{Z}_{\mathrm{mn}}^{\mathrm{T}} \mathrm{Z}_{\mathrm{mn}}, \quad \mathrm{Z}_{\mathrm{ki}}=\mathrm{r}_{\mathrm{ij}} \mathrm{Z}_{\mathrm{kj}}$. Знание значений $\mathrm{j}$-го столбца и значения $\mathrm{r}_{\mathrm{ij}}$ позволяет узнать значений $\mathrm{i}-$ го столбца матрицы $\mathrm{Z}_{\mathrm{mn}}$.

\section{3.Матрица $Z_{20,6}$ - преобразованный источник информации \\ Матрица $Z_{\mathrm{mn}}$ является попутчиком} (преобразованным) матрицы $\mathrm{C}_{\mathrm{nn}}$, содержащей индикаторы наличия информации, а информация преобразуется в знание, если применить когнитивное моделирование. Источников знаний, извлекаемых когнитивно, является матрица $\mathrm{C}_{\mathrm{nn}}$ значений «весов» и пренебрежимо малых «весов». Из них только весомые «веса» являются основными источниками цифровых знаний [2-7. А коэффициенты при значениях «весов» (при «коэффициентах

комбинационной пропорциональности» (ККП), [7]) являются измерителями степени изменчивости: их значения показывают (через свои значения $\left.\mathrm{c}_{\mathrm{ij}}, \mathrm{i}=1, \ldots, \mathrm{n}, \mathrm{j}=1, \ldots, \mathrm{n}\right) \quad$ насколько выражена непропорциональная изменчивость двух

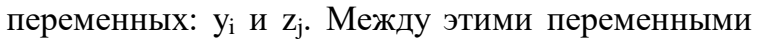
имеется корреляционная связь: $\operatorname{corr}\left(\mathrm{z}_{\mathrm{i}}, \mathrm{y}_{\mathrm{j}}\right)=\mathrm{c}_{\mathrm{ij}}$, элементы $\quad(\mathrm{y}, \mathrm{z})$-корреляций несимметрическую матрицу $\mathrm{C}_{\mathrm{nn}}$, являющуюся матрицей линейного преобразования $\mathrm{n} \mathrm{z}$ переменных в $\mathrm{n}$ у -переменных: $\mathrm{Y}_{\mathrm{mn}}=\mathrm{Z}_{\mathrm{mn}} \mathrm{C}_{\mathrm{nn}}$. Здесь одна переменная равна линейной комбинации $\mathrm{n}$ z-переменных: $\mathrm{y}_{\mathrm{ij}}=\mathrm{z}_{\mathrm{i} 1} \mathrm{c}_{1 \mathrm{j}}+\mathrm{z}_{\mathrm{i} 2} \mathrm{c}_{2 \mathrm{j}}+\ldots$ $+\mathrm{Z}_{\mathrm{in}} \mathrm{c}_{\mathrm{nj}}$, (не являются стандартизованными) другая переменная (z-переменная $\mathrm{z}_{\mathrm{i}}$ ) в формуле $\mathrm{c}_{\mathrm{ij}}=$ $\operatorname{corr}\left(\mathrm{z}_{\mathrm{i}}, \mathrm{y}_{\mathrm{j}}\right)$ является стандартизованной. Только после стандартизации одной из них: $\mathrm{y}_{\mathrm{i}} / \lambda_{\mathrm{j}}$ и $\mathrm{z}_{\mathrm{j}}(\mathrm{y}, \mathrm{z})-$ корреляция между ними- $\mathrm{c}_{\mathrm{ij}}=\operatorname{corr}\left(\mathrm{z}_{\mathrm{i}}, \mathrm{y}_{\mathrm{j}}\right)$ имеет тот смысл, который соответствует его определению.
Несимметрическая

$\mathrm{C}_{\mathrm{nn}}=(1 / \mathrm{m}) \mathrm{Z}_{\mathrm{mn}}^{\mathrm{T}}\left[\mathrm{Y}_{\mathrm{mn}} \Lambda^{(-1)}\right]$

коэффициентами

матрица

«весов» с

непропорциональных изменчивостей содержит значимые (значение элемента матрицы $\mathrm{C}_{\mathrm{nn}}$ имеет весомый «вес») и незначимые элементы (значение элемента матрицы $\mathrm{C}_{\mathrm{nn}}$ не имеет «веса», удовлетворяющего критерию весомости). Значимый элемент называем «вес» и используем его в качестве индикатора наличия содержательного смысла у zпеременной, значение $\mathrm{z}_{\mathrm{i} 2}$ которой умножается на значение значимого элемента: $\mathrm{Z}_{\mathrm{i} 1} \mathrm{c}_{1 \mathrm{j}}$. Весомый «вес» не единствен. Значения «весов» и весов из матриц $\mathrm{C}^{(\ell)}{ }_{n n}$ с номерами $\ell=1, \ldots, \propto$. Схематическое изображение ОМГК: $\Lambda=>(\mathrm{C}, \mathrm{R}, \mathrm{Y}, \mathrm{Z}$,$) , где \mathrm{Y}_{\mathrm{mn}}$ является решением О3 АГК, матрица $\mathrm{Z}_{\mathrm{mn}^{-}}$ ассоциированным решением О3 АГК. Фиксируется последовательность их - матриц, вычислений в ОМ ГК[1]: $\Lambda \rightarrow \mathrm{C}, \mathrm{R} \rightarrow \mathrm{Y} \rightarrow \mathrm{Z}$. Здесь моделируется матрица $\mathrm{C}$, потом вычисляются матрицы $\mathrm{R}=\mathrm{C} \Lambda \mathrm{C}^{\mathrm{T}}$., Y, Z.

4.Модельная многомерная выборка $\mathrm{X}^{\mathbf{0}} \mathbf{2 0 , 6}$

(с модельными значениями $\mathrm{n}=6$ признаков зерновой культуры)

Опишем шаги получения модельных значений $\mathrm{n}=6$ признаков зерновой культуры.

Имеем 2 целых числа. 2 вектора, 2 матрицы [11] $\mathbf{C}_{66}$ и $\Lambda_{66}=\operatorname{diag}(2.5117,1.592,1.18,0.462221049$, $0.173723201,0.08035575)$. В спектре $\Lambda_{66}$ не доминирующие его элементы $\lambda_{4}=0.462221049$, $\lambda_{5}=0.173723201, \lambda_{6}=0.08035575$ были оценены в работе [11]. Спектр $\Lambda_{66}$ является востановленным точно: его элементы удовлетворяют условиям 3-4. Матрица собственных векторов $\mathrm{C}_{66}$ доступна из публикаций и имеет вид (смотрите Таблицу 2).

Для моделирования матрицы $Z_{\mathrm{mn}}$ (Таблица 3), содержащей $m=20$ значений $Z_{k j}$ изменчивостей каждой из $\mathrm{n}$ z-переменных, $\mathrm{k}=1, \ldots, \mathrm{m}=20$; $\mathrm{n}=1, \ldots, \mathrm{n}=6, \quad$ реализуем ОМ ГК [1]: $\left(\mathrm{C}_{66}, \Lambda_{66}\right)=>\left(\mathrm{R}_{\mathrm{nn}}, \mathrm{U}^{(\mathrm{t})}{ }_{\mathrm{nn}}\right.$, где $\mathrm{Y}^{(\mathrm{t})}{ }_{\mathrm{mn}}=\mathrm{U}^{(\ell)}{ }_{\mathrm{nn}} \Lambda^{1 / 2}{ }_{66}, \mathrm{Z}^{(\mathrm{t})}{ }_{\mathrm{mn}}=$ $\left.\mathrm{Y}^{(\mathrm{t})}{ }_{\mathrm{mn}} \mathrm{C}^{\mathrm{T}}{ }_{\mathrm{nn}}\right), \quad \mathrm{t}=1, \ldots, \mathrm{k}_{\mathrm{t}}<\infty$. Матрицу $\mathrm{Z}_{\mathrm{mn}} \quad \mathrm{Z}-$ переменных и матрицу $\mathrm{Y}_{\mathrm{mn}}$ У-переменных, имеющих корреляционные матрицы, равные $\mathrm{I}_{\mathrm{nn}}=\operatorname{diag}(1, \ldots, 1): \quad \mathrm{Y}_{\mathrm{mn}}=\mathrm{U}_{\mathrm{nn}} \Lambda^{1 / 2}{ }_{66}$, $\mathrm{Z}^{(\mathrm{t})}{ }_{\mathrm{mn}}=\mathrm{Y}^{(\mathrm{t})}{ }_{\mathrm{mn}} \mathrm{C}^{\mathrm{T}}{ }_{\mathrm{nn}}=\mathrm{U}^{(\mathrm{t})}{ }_{\mathrm{nn}} \Lambda^{1 / 2}{ }_{66}$, $\mathrm{C}^{\mathrm{T}}{ }_{\mathrm{nn}},(1 / \mathrm{m}) \mathrm{U}^{(\mathrm{t}) \mathrm{T}}{ }_{\mathrm{mn}} \mathrm{U}^{(\mathrm{t})}{ }_{\mathrm{mn}}=\mathrm{I}_{\mathrm{nn}}$

Здесь $Z_{20,6}$-входной элемент решенной ранее ПЗ АГК [1,8]: $\mathrm{Z}_{131,6}=>\left(\mathrm{R}_{66}, \quad \mathrm{C}_{66}, \Lambda_{66}, \mathrm{Y}_{131,6}\right), \quad \mathrm{a}$ использованная нами известная матрица собственных векторов $\mathrm{C}_{66}$ (таблица 2) вычислена при решении прямой спектральной задачи (ПС3) диагонализации симметрической матрицы [1] $\mathrm{R}_{\mathrm{nn}}=>\left(\mathrm{C}_{\mathrm{nn}}, \Lambda_{\mathrm{nn}}\right)$. Матрица $\mathrm{R}_{\mathrm{nn}}$ потеряна, ее мы ниже восстанавливаем, $\Lambda_{\mathrm{nn}}$-была восстановлена из неполной: отсутствовали недоминирующие элементы $\lambda_{4}, \lambda_{5}, \lambda_{6}$. 


\begin{tabular}{|c|c|c|c|c|c|c|}
\hline \multirow{4}{*}{ Impact Factor: } & ISRA (India) & $=3.117$ & SIS (USA) & $=0.912$ & ICV (Poland) & $=6.630$ \\
\hline & ISI (Dubai, UAE & $=0.829$ & РИНЦ (Russia & $=0.156$ & PIF (India) & $=1.940$ \\
\hline & GIF (Australia) & $=0.564$ & ESJI (KZ) & $=8.716$ & IBI (India) & $=4.260$ \\
\hline & JIF & $=1.500$ & SJIF (Morocce & $=5.667$ & OAJI (USA) & $=0.350$ \\
\hline
\end{tabular}

Наши смоделированные цифровые данные для этих характеристик должны, как принято стандартами, отображены с соблюдением 6 форм округления (Таблица 4).

Наши работы по моделированию $\Lambda$-выборок, адекватных реальной выборке, проведены на высоком уровне. С применением ОМ ГК [1]. Одна из $\Lambda$-выборок $\mathrm{Z}^{(\mathrm{t})}$ m20,6 -ассоциированных решений O3 АГК преобразована в матрицу (таблица 3) модельных данных, адекватна реальной многомерной выборке [12], с заданными (известными из публикации [11]) значениями выборочных средних $\quad \mathrm{x}^{\mathrm{cp}}{ }_{16}=(45.75,106.1,19.15$, $1.1367,40.45,27.65)$ и выборочных стандартных отклонений $\mathrm{s}_{16}=\operatorname{diag}(4.4931,7.5425,1.711,0.1532$, 5.0742,2.7798), вычисленных по матрице реальных данных размерности $20 \times 6$.

Номерам столбцов соответствуют названия свойств (признаков) зерновой культуры [11]: №1 - длина стебля, №2- длина колоска, №3- число колосков в стебле, №4- вес одного зернышка в колоске, №5- число зерен в колоске, №6-вес 1000 зерен (в условных единицах). Номера строк матрицы данных соответствуют названиям географических районов, на землях которых выращивались селекционерами новые сорта зерновой культуры.

Практика-селекционера не удовлетворяют такие формы округления чисел 50.6465 (№1), 101.1557(№2), 19.5968(№3), $\quad$ 1.1575(№4), 38.9714(№5), 30.4524(№6) не удовлетворяют. Длина стебля 101,1557 сантиметра (№2) не может быть измерена пи помощи линейки, скорее всего длина стебля равна 101,2 сантиметра. Таких превышений возможностей шкал измерительных механизмов в элементах Таблицы 3 очень много.

Таблица 3. $\Lambda$-выборка $\left(\mathrm{X}^{0}{ }_{20,6}\right)$ модельных значений 6 признаков зерновой культуры

\begin{tabular}{|l|l|l|l|l|l|l|l|}
\hline COLUMN & № & 1 & 2 & 3 & 4 & 5 & 6 \\
\hline ROW & 1 & 50,6465 & 101,1557 & 19,5968 & 1,1575 & 38,9714 & 30,4524 \\
\hline ROW & 2 & 53,7863 & 97,7753 & 16,8319 &, 8466 & 46,0738 & 27,9270 \\
\hline ROW & 3 & 39,9812 & 112,8104 & 21,6241 & 1,3718 & 34,7682 & 28,6621 \\
\hline ROW & 4 & 45,4231 & 110,1430 & 19,9762 &, 8581 & 40,9447 & 28,9168 \\
\hline ROW & 5 & 52,0438 & 97,5934 & 17,0666 & 1,2870 & 38,1134 & 26,5059 \\
\hline ROW & 6 & 46,3259 & 96,1398 & 18,8887 & 1,2359 & 29,8642 & 23,6580 \\
\hline ROW & 7 & 41,2376 & 114,2718 & 19,7418 & 1,1914 & 39,4097 & 26,0280 \\
\hline ROW & 8 & 42,1696 & 114,8493 & 18,3934 & 1,1731 & 36,8668 & 23,7165 \\
\hline ROW & 9 & 45,6037 & 106,3635 & 21,4545 & 1,2996 & & 34,4225 \\
\hline ROW & 10 & 51,3892 & 99,7731 & 16,5554 & 1,2323 & 46,3970 & 27,8017 \\
\hline ROW & 11 & 52,4501 & 91,5348 & 17,8889 & 1,0804 & 36,3417 & 26,5476 \\
\hline ROW & 12 & 48,9728 & 100,7432 & 19,8068 & 1,2153 & 43,2183 & 32,3054 \\
\hline ROW & 13 & 44,0212 & 115,6814 & 18,6571 &, 8432 & 44,9187 & 26,5810 \\
\hline ROW & 14 & 44,0952 & 100,5764 & 16,8484 & 1,0230 & 37,9575 & 19,9715 \\
\hline ROW & 15 & 42,2807 & 105,5790 & 20,8449 & 1,1769 & 41,2844 & 28,9277 \\
\hline ROW & 16 & 39,6350 & 110,0727 & 22,2713 & 1,3195 & 38,9900 & 30,2454 \\
\hline ROW & 17 & 40,2877 & 108,8376 & 20,2829 & 1,1485 & 41,8057 & 28,0110 \\
\hline ROW & 18 & 41,8710 & 120,8311 & 20,2992 & 1,2266 & 43,3891 & 29,7066 \\
\hline ROW & 19 & 43,1701 & 106,2760 & 19,2713 & 1,0573 & 41,3743 & 26,9380 \\
\hline ROW & 20 & 49,6094 & 110,9924 & 16,6999 &, 9899 & 53,8884 & 29,3848 \\
\hline
\end{tabular}

\section{5.Модельная многомерная выборка $\mathbf{X}^{\mathbf{0}} \mathbf{2 0 , 6}$}

(с реалистичными значениям $\mathrm{n}=6$ признаков зерновой культуры)

Рассмотрим основные моменты придания реалистичности числовым значениям 6 признаков зерновой культуры. В расчетах округления наших модельных данных удаленной конечной целью является расчет показателя «урожайность с одного гектара пахотной земли». Этот показатель является наиважнейшим, мы стремились выявить регулируемые параметры (), учет которых позволяет степень неопределенности на значения 6 z-переменных в формуле $\mathrm{x}_{\mathrm{ij}}{ }^{\circ}=\mathrm{z}_{\mathrm{ij}} \mathrm{s}_{\mathrm{j}}+\mathrm{x}_{\mathrm{j}}{ }^{\mathrm{cp}}$, где доминирующий вклад в сумму вносит слагаемое $\mathrm{x}_{\mathrm{j}}{ }^{\mathrm{cp}}$, а второе слагаемое $\mathrm{z}_{\mathrm{ij}} \mathrm{S}_{\mathrm{j}}$, есть случайное число $\mathrm{Z}_{\mathrm{ij}}$ с постоянным множителем $\mathrm{s}_{\mathrm{j}}$. Если значения чисел $\mathrm{x}_{\mathrm{j}}{ }^{\mathrm{cp}}$ и $\mathrm{Z}_{\mathrm{ij}} \mathrm{S}_{\mathrm{j}}$,Удовлетворяют форме округления, то значение числа может не удовлетворять той же форме округления. В нашем случае из видимых знаков в числах 6 средних видно, что они не удовлетворяют формам округления ни одно из двух слагаемых. Формы округления важны для практических работников агробизнеса. При наличии их скептического отношения к «производителям мешков с синусами». Заметим: значения функции sin материализуются, если они 


\begin{tabular}{|c|c|c|c|c|c|c|}
\hline \multirow{4}{*}{ Impact Factor: } & ISRA (India) & $=3.117$ & SIS (USA) & $=0.912$ & ICV (Poland) & $=6.630$ \\
\hline & ISI (Dubai, UAE & $=0.829$ & РИНЦ (Russia & $=0.156$ & PIF (India) & $=1.940$ \\
\hline & GIF (Australia) & $=0.564$ & ESJI (KZ) & $=8.716$ & IBI (India) & $=4.260$ \\
\hline & JIF & $=1.500$ & SJIF (Morocce & $=5.667$ & OAJI (USA) & $=0.350$ \\
\hline
\end{tabular}

удовлетворяют формам округления. Цель: отказ от прямых линий и углов (в архитектуре) в пользу более естественных, «природных» линий достигается моделированием по модельным линиям из точек с координатами (y,z), удовлетворяющих формуле $\mathrm{z}=\mathrm{kx} \sin (\mathrm{y} / \mathrm{a})$ и материализуются в виде поверхности Гауди. Мы тоже применяем символы z,k,x,sin,y,a, но наши модельные точки не материализуются в поверхности, но используются в IT-технологий для достижения конкретной, измеримой, достижимой, актуальной, ограниченной сроком цели, сформулированной в заглавии статьи. Для существующих методик подсчета урожайности, учитывающих различные факторы.

Придадим черты реалистичности модельным числам из таблицы 3. На практике проводились реальные измерения при помощи реальных устройств.

Длина стебля (№1) измерялась в сантиметрах с точностью до 2-х знаков после запятой: по формату F5.2, длина колоска (№2) - в сантиметрах с точностью до 1 знака после запятой:: по формату F5.1,число колосков в стебле (№3) - измерялось в целых числах: по формату $\mathrm{F} 2.0$, как двузначное целое число, вес одного зернышка в колоске (№4)- в граммах высокоточным измерителем веса с точностью до 4-х знаков после запятой: по формату F6.4,число зерен в колоске (№5)- измерялось в целых числах: по формату F2.0, как двузначное целое число, вес 1000 зерен (№6)- в граммах с точностью до 4-х знаков после запятой:: по формату F9.4.

Измерения преследуют разные ежегодно достигаемые цели. Одна из целей-практический расчет урожайности зерна, выращенного и прошедшего полный цикл превращений до готовой продукции разных видов. Веса на входе должны совпадать с весами на выходе. В цепочке превращений зерна с поля до макарон на прилавке должны существуют разные методики определения весов. Для 1 кг макарон на прилавке магазина существует свой виртуальный вес одного зернышка, отличающийся от веса, измеренного в поле. Если мы напишем «значение этого признака №6 равно значению признака №4, умноженного на 1000», то мы будем неправы.

Значение признака №3- число колосков в стебле, №4- вес одного зернышка в колоске, №5число зерен в колоске) и среднего веса 1000 зерен, при котором в те периоды, когда отдельные элементы еще не сформировались, они принимаются в размерах, соответствующих средним многолетним. Упомянем лишь о методиках ученых кафедры статистики Тимирязевской сельскохозяйственной академии по прогнозу урожая на основе учета агротехнических и метеорологических факторов урожайности.

Пока мы знаем лишь ожидаемое значение признака №6 (вес 1000 зерен) равное 27.65 условных единиц. Стандарт на зерно пшеницы, предназначенное для продовольственных и кормовых целей, выработки комбикормов и устанавливает метод определения веса зернышка регулирует ГОСТ 13586.3-2015 Зерно. Правила приемки и методы отбора проб. Этот ГОСТ предписывает, например, «результаты вычисляют с точностью до второго десятичного знака и округляют до первого десятичного знака».

Имитации округлений 6 признаков зерновой культуры в поле с применением моделей описаны выше. Эти модели очень точны, широко апробированы $[1,12,13]$.

Таблица 4.

\begin{tabular}{|l|l|l|l|l|l|l|}
\hline $\begin{array}{c}\text { № } \\
\Pi \backslash \Pi\end{array}$ & \multicolumn{6}{l|}{ Реалистичные значения 6 признаков зерновой культуры } \\
\hline & 1 & 2 & 3 & 4 & 5 & 6 \\
\hline 1 & 50,65 & 101,2 & 20 & 1,1575 & 39 & 30,4524 \\
\hline 2 & 53,79 & 97,78 & 17 & 0,8466 & 46 & 27,927 \\
\hline 3 & 39,98 & 112,8 & 22 & 1,3718 & 35 & 28,6621 \\
\hline 4 & 45,42 & 110,1 & 20 & 0,8581 & 41 & 28,9168 \\
\hline 5 & 52,04 & 97,59 & 17 & 1,287 & 38 & 26,5059 \\
\hline 6 & 46,33 & 96,14 & 19 & 1,2359 & 30 & 23,658 \\
\hline 7 & 41,24 & 114,3 & 20 & 1,1914 & 39 & 26,028 \\
\hline 8 & 42,17 & 114,8 & 18 & 1,1731 & 37 & 23,7165 \\
\hline 9 & 45,6 & 106,4 & 21 & 1,2996 & & 34,4225 \\
\hline 10 & 51,389 & 99,773 & 17 & 1,2323 & 46 & 27,8017 \\
\hline 11 & 52,45 & 91,535 & 18 & 1,0804 & 36 & 26,5476 \\
\hline 12 & 48,973 & 100,74 & 20 & 1,2153 & 43 & 32,3054 \\
\hline 13 & 44,021 & 115,68 & 19 & 0,8432 & 45 & 26,581 \\
\hline
\end{tabular}




\begin{tabular}{|c|c|c|c|c|c|c|}
\hline \multirow{4}{*}{ Impact Factor: } & ISRA (India) & $=3.117$ & SIS (USA) & $=0.912$ & ICV (Poland) & $=6.630$ \\
\hline & ISI (Dubai, UAE & $=0.829$ & РИНЦ (Russia & $=0.156$ & PIF (India) & $=1.940$ \\
\hline & GIF (Australia) & $=0.564$ & ESJI (KZ) & $=8.716$ & IBI (India) & $=4.260$ \\
\hline & JIF & $=1.500$ & SJIF (Morocce & $=5.667$ & OAJI (USA) & $=0.350$ \\
\hline
\end{tabular}

\begin{tabular}{|l|l|l|l|l|l|l|}
14 & 44,095 & 100,58 & 17 & 1,023 & 38 & 19,9715 \\
\hline 15 & 42,281 & 105,58 & 21 & 1,1769 & 41 & 28,9277 \\
\hline 16 & 39,635 & 110,07 & 22 & 1,3195 & 39 & 30,2454 \\
\hline 17 & 40,288 & 108,84 & 20 & 1,1485 & 42 & 28,011 \\
\hline 18 & 41,871 & 120,83 & 20 & 1,2266 & 43 & 29,7066 \\
\hline 19 & 43,17 & 106,28 & 19 & 1,0573 & 41 & 26,938 \\
\hline 20 & 49,609 & 110,99 & 17 & 0,9899 & 54 & 29,3848 \\
\hline
\end{tabular}

Для выявленных из ГОСТ форм округления применим форматирование столбцов в ЭТ Excel. Электронная таблица Excel позволяет визуализировать числа в столбце в нужном формате. Проблема состоит в том, чтобы знать стандарты измерения наших 6 показателей.

На гистограммах из [1] и на гистограммах наших 1-мерных переменных видны одинаковости чисел точек в интервалах осей интервалов гистограмм модельной и реальной выборок. Это-обратная задача: при неизвестной многомерной функции распределения случайного вектора $\xi=\left(\xi_{1}, \ldots, \xi_{6}\right)$ найти 1-мерное распределение каждой из 6 зависимых компонент $\xi_{1}, \ldots, \xi_{6}$. Решения этой обратной задачи нет [13].

Описанных выше свойств достаточно, чтобы, используя этот спектр с новыми не доминирующими элементами, можно моделировать $[1,11,12,13]$ имеющие общий спектр $\Lambda$-выборки $Z^{(t)}{ }_{m n}, \mathrm{t}=1, \ldots, \mathrm{k}_{\mathrm{t}}$.

Наши модельные $\Lambda$-выборки, воспроизведенные по восстановленному спектру, наравне с $\Lambda$-выборками по «реальному» спектру, будут использованы при решении задач «извлечения цифровых знаний» из цифровых данных из разных предметных областей, организованных в виде таблицы объект-свойства, например, средствами когнитивного моделирования [2-6,11].

\section{Заключение}

Мы применили формы округления форматирование столбцов в ЭТ Excel. Электронная таблица Excel позволяет визуализировать числа в столбце в нужном формате. Проблема состоит в том, чтобы знать стандарты измерения наших 6 показателей. Рассмотрели основные математические (ПЗ АГК,О3 АГК,ПСЗ,ОСЗ, оптимизационные задачи), статистические модели (ПМ ГК, ОМ ГК) придания модельной адекватности числовым значениям 6 признаков зерновой культуры. Достигнута модельная и гистограммная адекватности значений $6 \mathrm{z}$-переменных [ ] и $6 \mathrm{x}^{0}$ переменных, линейно зависящих от $\mathrm{z}$ переменных.

В моделировании значений признаков, измеряемых в 6 разных единицах измерения и масштабах, применили стандартизованные выборки из ОМ ГК, достигли реалистичных значений 6 признаков зерновой культуры (Таблица 4). Практика-селекционера удовлетворили отсутствие превышений возможностей шкал измерительных механизмов в элементах Таблицы 4.

Мы в применяемых моделях выявили регулируемые параметры, учет которых позволил достичь через высококачественные датчики равномерно распределенных чисел, достигается высокая степень неопределенности на 20 значений 6 z-переменных в формуле $\mathrm{x}_{\mathrm{ij}}{ }^{\circ}=\mathrm{z}_{\mathrm{ij}} \mathrm{s}_{\mathrm{j}}+\mathrm{x}_{\mathrm{j}}{ }^{\mathrm{cp}}$, удовлетворяют форме округления.

Таблица 4 демонстрирует все видимые цифровые знаки, придание реалистичности значениям признаков зерновой культуры в модельной $\Lambda$-выборке. Таблица 4 не вызывает скептического отношения к $\Lambda$-выборке.

\section{References:}

1. Zhanatauova, S. U. (2013). Obratnaya model' glavnykh komponent. (p.201). Almaty: Kazstatinform.

2. Zhanatauov, S. U. (2018). Inverse spectral problem. Int. Scientific Journal Theoretical
\&Applied Science, №12(68), 101-112. www.tscience.org

3. Zhanatauov, S. U. (2016). Modeling eigenvectors with given the values of their indicated components. International Scientific 


\begin{tabular}{llllll} 
& ISRA (India) $=\mathbf{3 . 1 1 7}$ & SIS (USA) $=\mathbf{0 . 9 1 2}$ & ICV (Poland) & $\mathbf{= 6 . 6 3 0}$ \\
Impact Factor: & ISI (Dubai, UAE) $=\mathbf{0 . 8 2 9}$ & PUHЦ (Russia) $=\mathbf{0 . 1 5 6}$ & PIF (India) & $=\mathbf{1 . 9 4 0}$ \\
& GIF (Australia) $=\mathbf{0 . 5 6 4}$ & ESJI (KZ) & $\mathbf{8 . 7 1 6}$ & IBI (India) & $=\mathbf{4 . 2 6 0}$ \\
& JIF & $\mathbf{1 . 5 0 0}$ & SJIF (Morocco) $=\mathbf{5 . 6 6 7}$ & OAJI (USA) & $\mathbf{0 . 3 5 0}$ \\
\hline
\end{tabular}

Journal Theoretical \&Applied Science, №11, vol.43, pp.107-119. www.T-science.Org

4. Zhanatauov, S. U. (2018). Inverse spectral problem with indicated values of components of the eigenvectors. Int. Scientific Journal Theoretical\&Applied Science, №1 1(67), pp.359370. www.T-science.Org

5. Zhanatauov, S. U. (2018). Model of digitalization of the validity indicators and of the measurable indicators of the enterprise. Int.Scien.Jour. "Theoretical \&Applied Science", № 9(65): pp.315-334. www.T-Science.org

6. Zhanatauov, S. U. (2018). Model of digitalization of indicators of individual consciousness. ISJ "Theoretical \&Applied Science”, №6(62): pp.101-110. www.tscience.org

7. Zhanatauov, S. U. (2019). A matrix of values the coefficients of combinational proportionality. Int. Scientific Journal Theoretical \&Applied Science, 71№3, 401-419. www.t-science.org

8. Hotelling, H. (1933). Analysis of a complex of statistical variables into principal components. J.Educ. Psych., v.24, pp. 417,441,498-520.

9. Zhanatauov, S. U. (2017). Optimization problem of modeling missing elements of the spectrum of the correlation matrix. International scientific journal Theoretical \&Applied Science, №10, vol.54, pp.189-198. www.t-science.org

10. Zhanatauov, S. U. (2017). The optimization problem with linearized equations f-parameters (f1,f2,f3,f4,f5,f6)-spectrum. International scientific journal Theoretical \&Applied Science, №1 1, vol.55, pp.251-267. www.t-science.org

11. Zhanatauov, S. U. (2017). Modelirovanie mnogomernykh vyborok znacheniy priznakov zernovoy kul'tury. "II mezhdun. nauchnoprakt.konf. «Evropa i tyurkskiy mir: nauka, tekhnika i tekhnologii". Izmir (Turtsiya), 29-31 maya 2017. www.regionacadem.org

12. Zhanatauov, S. U. (2016). Model and histogram to adequacy of variables $(\mathrm{C}, \Lambda)$ - samples and real multi-dimensional sample. International Scientific Journal Theoretical \&Applied Science, № 11, vol. 4, pp. 53-61. www.T-science.Org

13. Zhanatauov, S. U. (2014). The (C, $\Lambda, Y)$-sample is adequate to real multidimensional sample. Proced. Int. conf.. "Leadership in Education, Business and Culture". 25 apriel 2014, AlmatySeatle, ICET USA. Leadership Iternational Conference "Leadership on Education, Business and Culture». pp.151-155 